\title{
A SPECIAL EXTENSION OF WIEFERICH'S CRITERION
}

\author{
PETR CIKÁNEK
}

ABstract. The following theorem is proved in this paper: "If the first case of Fermat's Last Theorem does not hold for sufficiently large prime $l$, then

$$
\sum_{x} x^{l-2}\left[\frac{k l}{N}<x<\frac{(k+1) l}{N}\right] \equiv 0 \quad(\bmod l)
$$

for all pairs of positive integers $N, k, N \leq 94,0 \leq k \leq N-1$." The proof of this theorem is based on a recent paper of Skula and uses computer techniques.

\section{INTRODUCTION}

The first case of Fermat's Last Theorem states that for each odd prime $l$ the equation

$$
x^{l}+y^{l}+z^{l}=0
$$

has no integral solution $x, y, z$ with $l \nmid x y z$.

One of many methods investigating this problem was introduced by $A$. Wieferich. This method is connected with the Fermat quotients $q_{l}(a)$,

$$
q_{l}(a)=\frac{a^{l-1}-1}{l}
$$

defined for each integer $a$ such that $a$ is not divisible by $l$.

Let us assume in this paragraph that $l$ is an odd prime which does not satisfy the first case of Fermat's Last Theorem.

In 1909, Wieferich [7] published the following important result:

$$
q_{l}(2) \equiv 0 \quad(\bmod l) .
$$

Many mathematicians have extended this Wieferich criterion. The latest result is due to A. Granville and B. Monagan [1] and states $q_{l}(p) \equiv 0(\bmod l)$ for each prime $p$ such that $p \leq 89$.

These considerations have been generalized by L. Skula. He studied the sums

$$
s(k, N)=\sum_{x} x^{l-2}\left(\frac{k l}{N}<x<\frac{(k+1) l}{N}\right)
$$

Received by the editor May 6, 1991 and, in revised form, November 25, 1991 and November $5,1992$.

1991 Mathematics Subject Classification. Primary 11D41.

Key words and phrases. The first case of Fermat's Last Theorem, Fermat quotient, Bernoulli numbers, Bernoulli polynomials. 
for integers $N, k, 1 \leq N \leq l-1,0 \leq k \leq N-1$. These sums are connected with the Fermat quotients by a formula introduced essentially by M. Lerch [2]:

$$
q_{l}(N) \equiv N^{l-2} \sum_{k=0}^{N-1} k s(k, N) \quad(\bmod l) .
$$

Skula [4] proved

$$
s(k, N) \equiv 0 \quad(\bmod l), \quad 0 \leq k \leq N-1,
$$

for each $N \in\{2,3, \ldots, 10\} \cup\{12\}$.

In this paper, Skula's result is improved for integers $N \leq 94$ (Main Theorem 3.2), but only for sufficiently large primes $l$.

Remark. It is easy to prove that the statement

$$
s(k, N) \equiv 0 \quad(\bmod l), \quad 0 \leq k \leq N-1,
$$

is equivalent to the statement

$$
B_{l-1}\left(\frac{j}{N}\right)-B_{l-1} \equiv 0 \quad(\bmod l), \quad 0 \leq j \leq N,
$$

where $B_{n}, B_{n}(x)$ are the $n$th Bernoulli number and Bernoulli polynomial, respectively. Therefore, our result implies that the polynomial $B_{l-1}(t)-B_{l-1}$ has at least $1+\sum_{N=1}^{94} \varphi(N)=2703$ distinct zeros modulo $l$ for sufficiently large prime $l$, where $l$ does not satisfy the first case of Fermat's Last Theorem.

\section{BASIC NOTIONS AND ASSERTIONS}

We will assume in this section that there is an odd prime $l$ which does not satisfy the first case of Fermat's Last Theorem, briefly (FLTI) $l$ fails; i.e., there exist integers $x, y, z$ such that

$$
x^{l}+y^{l}+z^{l}=0, \quad l \nmid x y z .
$$

1.1. Definition. Let $\tau_{1}, \ldots, \tau_{6}$ denote the integers satisfying

$$
\begin{array}{lll}
x \tau_{1} \equiv-y(\bmod l), & x \tau_{3} \equiv-z(\bmod l), & y \tau_{5} \equiv-z(\bmod l), \\
y \tau_{2} \equiv-x(\bmod l), & z \tau_{4} \equiv-x(\bmod l), & z \tau_{6} \equiv-y(\bmod l) .
\end{array}
$$

The definition of $\tau_{1}, \ldots, \tau_{6}$ implies

1.2. Lemma. The integers $\tau_{1}, \ldots, \tau_{6}$ satisfy the following congruences:

$$
\begin{gathered}
\tau_{1} \tau_{2} \equiv \tau_{3} \tau_{4} \equiv \tau_{5} \tau_{6} \equiv 1 \quad(\bmod l), \\
\tau_{1}+\tau_{3} \equiv \tau_{2}+\tau_{5} \equiv \tau_{4}+\tau_{6} \equiv 1 \quad(\bmod l), \\
0 \not \equiv \tau_{i} \not \equiv 1 \quad(\bmod l), \quad 1 \leq i \leq 6 .
\end{gathered}
$$

According to the results of Pollaczek ([3], See [1, Lemma 15]) we have

1.3. Lemma. Let $r_{1}, \ldots, r_{6}$ denote the orders of the integers $\tau_{1}, \ldots, \tau_{6} \bmod l$. Then $r_{1}=r_{2}, r_{3}=r_{4}, r_{5}=r_{6}$, and each of the products $r_{1} r_{3}, r_{3} r_{5}, r_{1} r_{5}$ is greater than or equal to

$$
\frac{3 \log (l)}{\log \left(\frac{1+\sqrt{5}}{2}\right)} \text {. }
$$


1.4. Definition. Pollaczek introduced a matrix $A_{s}(t)$ of size $2 \varphi(s) \times \varphi(s) \quad(\varphi$ Euler's function) for integers $s \geq 2$ and variable $t$ in [3]. Let $r(s, t)$ denote the rank of the matrix $A_{s}(t)$ over the finite $\mathrm{Z} / l \mathbf{Z}$.

According to the results from [1, Table 1] (see also [4, 5.1.1]) we obtain

1.5. Lemma. Let $s, t$ be integers, $2 \leq s \leq 46$ and the order of $t$ modulo $l$ be greater than 44 . Then $r(s, t)=\varphi(s)$.

1.6. Definition. Skula ([4, Definition 4.13]) has introduced the following square matrix $D_{N}=D_{N}(t)$ of order $\frac{\varphi(N)}{2}$ for integers $N \geq 3$ and variable $t$ by the formula

$$
\begin{aligned}
D_{N}=D_{N}(t)=\left[t^{z(u, v)-1}+t_{u, v}^{N-1-z(u, v)}\right], \\
1 \leq u, v \leq \frac{N}{2}, \operatorname{gcd}(u, N)=\operatorname{gcd}(v, N)=1,
\end{aligned}
$$

where $z(u, v)$ is the integer such that $1 \leq z(u, v) \leq N-1, v \equiv u z(u, v)$ $(\bmod N)$.

Let us denote $d_{N}(t)=\operatorname{det} D_{N}(t)$.

The next theorem follows from Skula's results ([4, Main Theorem 4.14, 5.4.2]).

1.7. Theorem. Let $N$ be an integer, $N \geq 2, \frac{(N-2)(N-1)}{2}<l$, and $\tau_{1}, \ldots, \tau_{6}$ be the integers from 1.1. Assume that there exists $1 \leq a \leq 6$ such that the following conditions are satisfied:

(a) $d_{M}\left(\tau_{a}\right) \not \equiv 0(\bmod l)$ for each integer $M \geq 3, M \mid N$;

(b) $r\left(s, \tau_{a}\right)=\varphi(s)$ for each integer $s, 2 \leq s<\frac{N}{2}$.

Then $s(k, N) \equiv 0(\bmod l)$ for each $0 \leq k \leq N-1$.

\section{SOME AUXILIARY STATEMENTS}

2.1. Lemma. Let $p$ be a prime, $f(t), g(t)$ be polynomials over $\mathbf{Z}$, the leading coefficients of which are not divisible by $p$. If $f, g$ are relatively prime over the finite field $\mathbf{Z} / p \mathbf{Z}$, then $f, g$ are relatively prime over $\mathbf{Q}$.

Proof. It is sufficient to prove that $\operatorname{gcd}(f, g)$ over $\mathbf{Z}$ is a constant. Assume on the contrary that there exist polynomials $h, u, v$ over $\mathbf{Z}$ such that

$$
f=h u, \quad g=h v, \quad \operatorname{deg}(h)>0 .
$$

We can consider $f, g, h, u, v$ as polynomials over $\mathbf{Z} / p \mathbf{Z}$. Their degrees do not change because $p$ does not divide the leading coefficients of these polynomials. Then the equation (1) holds also over $\mathbf{Z} / p \mathbf{Z}$, and this is a contradiction.

2.2. Theorem. Let $m$ be a positive integer. There is an integer $L_{0}=L_{0}(m)$ with the following property:

Let $l>L_{0}$ be a prime for which $(F L T I)_{l}$ fails. Then there exist two different integers $a, b, 1 \leq a, b \leq 6$, such that

$$
\tau_{a}+\tau_{b} \equiv 1 \quad(\bmod l), \quad r_{a}>m, r_{b}>m,
$$

where $r_{a}, r_{b}$ are the orders of the integers $\tau_{a}, \tau_{b}$ modulo $l$. 
Proof. Let $L_{0}$ be the smallest integer greater than

$$
\left(\frac{1+\sqrt{5}}{2}\right)^{m^{2} / 3} \text {. }
$$

The proof then easily follows from Pollaczek's Lemmas 1.3. and 1.2.

2.3. Theorem. Let $N$ be an integer, $2 \leq N \leq 94, d(t)$ be any common multiple of the polynomials $d_{M}(t), 3 \leq M, M \mid N$. Let $g(t)$ be a polynomial such that:

(a) $g(t)$ is a product of some cyclotomic polynomials,

(b) $g(t) \mid d(t)$ over the ring $\mathbf{Z}[t]$ (we allow $g(t)=1$ ).

Let the polynomial $f(t)=\frac{d(t)}{g(t)}$ satisfy

$$
\operatorname{gcd}(f(t), f(1-t))=1 \quad \text { over } \mathbf{Q} .
$$

Then there exists a positive integer $L$ such that

$$
s(k, N) \equiv 0 \quad(\bmod l), \quad 0 \leq k \leq N-1,
$$

for each prime $l>L$ for which $(F L T I)$, fails.

Proof. Suppose $f(t), f(1-t)$ are relatively prime over the field $\mathbf{Q}$. Then there exist an integer $c$ and integral polynomials $u(t), v(t)$ such that

$$
f(t) u(t)+f(1-t) v(t)=c .
$$

Let $c$ be the smallest integer with this property.

Let us put $n_{0}=\max \left\{n, \Phi_{n}(t) \mid g(t)\right\} \quad\left(\Phi_{n}\right.$ is the $n$th cyclotomic polynomial), $m=\max \left\{n_{0}, 45\right\}, L_{0}=L_{0}(m)$ the integer from 2.2.

Let $l$ be a prime, $l>L_{0}, l \nmid c$, for which (FLTI), fails. According to 2.2 there exist different integers $a, b, 1 \leq a, b \leq 6$, such that

$$
\tau_{a}+\tau_{b} \equiv 1 \quad(\bmod l), \quad r_{a}>m, r_{b}>m .
$$

By $(3)$ we have $f\left(\tau_{a}\right) \not \equiv 0(\bmod l)$ or $f\left(\tau_{b}\right) \not \equiv 0(\bmod l)$. Therefore, we can assume

$$
f\left(\tau_{a}\right) \not \equiv 0 \quad(\bmod l) .
$$

Since $r_{a}>m \geq n_{0}$, we have

$$
\Phi_{n}\left(\tau_{a}\right) \not \equiv 0 \quad(\bmod l), \quad 1 \leq n \leq n_{0},
$$

and it follows that

$$
g\left(\tau_{a}\right) \not \equiv 0 \quad(\bmod l) .
$$

Putting (4) and (5) together, we obtain

$$
f\left(\tau_{a}\right) g\left(\tau_{a}\right)=d\left(\tau_{a}\right) \not \equiv 0 \quad(\bmod l) ;
$$

therefore,

$$
d_{M}\left(\tau_{a}\right) \not \equiv 0 \quad(\bmod l)
$$

for all integers $M, 3 \leq M \leq N, M \mid N$.

We can see that the integer $a$ satisfies the first condition of Theorem 1.7. The second condition is satisfied according to 1.5 . The proof now immediately follows from Theorem 1.7. 
What follows is useful for practical computer calculation. Instead of dealing with polynomials $d_{M}(t)=\operatorname{det} D_{M}(t)$, it allows to work with polynomials of lower degrees. These assertions follow from Washington's book [6, (4.5.26)]. For the convenience of readers we include proofs of these assertions.

Let $\chi$ be an even Dirichlet's character $\bmod M$. Let $f_{\chi}(t)$ be a polynomial of form

$$
f_{\chi}(t)=\sum_{i} \chi(i) t^{i-1}, \quad 1 \leq i \leq M, \operatorname{gcd}(i, M)=1 .
$$

2.4. Lemma. Let $M$ be an integer, $M \geq 3$. Then

$$
\operatorname{det} D_{M}(t)= \pm \prod_{\chi} f_{\chi}(t)
$$

where the product is over all even Dirichlet's characters $\bmod M$.

Proof. Let $\langle\alpha\rangle$ denote the fractional part of a real number $\alpha$. It is easy to see that

for each integer $x$.

$$
x \equiv M\left\langle\frac{x}{M}\right\rangle \quad(\bmod M)
$$

According to 1.6 we have

$$
\begin{aligned}
& D_{M}(t)=\left[t^{-1}\left(t^{z(u, v)}+t^{M-z(u, v)}\right)\right]_{v, u}, \quad 1 \leq u, v \leq \frac{M}{2}, \\
& \operatorname{gcd}(u, M)=\operatorname{gcd}(v, M)=1, \\
& \quad 1 \leq z(u, v) \leq M-1, v \equiv u z(u, v)(\bmod M) .
\end{aligned}
$$

Putting $i \equiv \pm u^{-1}(\bmod l)$, so that $1 \leq i \leq \frac{M}{2}$, we get

$$
d_{M}(t)= \pm t^{-\varphi(M) / 2} \operatorname{det} A,
$$

where $A$ is a matrix of the form

$A=\left[t^{M\langle i v / M\rangle}+t^{M\langle-i v / M\rangle}\right]_{i, v}, \quad 1 \leq i, v \leq \frac{m}{2}, \operatorname{gcd}(i, M)=\operatorname{gcd}(v, M)=1$.

Now it is sufficient to show that

$$
\operatorname{det} A= \pm t^{\varphi(M) / 2} \prod_{\chi} f_{\chi}(t),
$$

where the product is over all even characters $\bmod M$.

Let $B$ be the square matrix

$$
B=[\chi(i)]_{\chi, i},
$$

$\chi$ an even Dirichlet's character $\bmod M, 1 \leq i \leq \frac{M}{2}, \operatorname{gcd}(i, M)=1$. It is easy to prove that this matrix is nonsingular (see, e.g., Van der Waerden [5, $\S \S 124-126])$, and we have

$$
B A=\left[\sum_{i} \chi(i) t^{M\langle i v / M\rangle}\right]=\left[\chi^{-1}(v) \sum_{i} \chi(i) t^{i}\right]_{\chi, v}
$$

$$
(1 \leq i \leq M, \operatorname{gcd}(i, M)=1) \text {; }
$$

hence

$$
\operatorname{det} B \operatorname{det} A= \pm t^{\varphi(M) / 2}\left(\prod_{\chi} f_{\chi}(t)\right) \operatorname{det} B .
$$

This completes the proof. 
2.5. Lemma. Let $\chi$ be an even character $\bmod M$ of order $n \geq 1$. Then the polynomial

$$
F_{\chi}(t)=\prod_{a} f_{\chi^{a}}(t), \quad 1 \leq a \leq n, \operatorname{gcd}(a, n)=1,
$$

is a polynomial with integer coefficients.

Proof. The polynomial $f_{\chi}(t)$ is polynomial over the field $\mathbf{Q}\left(\xi_{n}\right), \xi_{n}=e^{2 \pi i / n}$. Let us consider the Galois group $G$ of the extension $\mathbf{Q}\left(\xi_{n}\right) / \mathbf{Q}$. It is well known that

$$
G=\left\{\sigma_{s}, s \in \mathbf{Z}, \quad 1 \leq s \leq n, \operatorname{gcd}(s, n)=1, \sigma_{s}\left(\xi_{n}\right)=\xi_{n}^{s}\right\} .
$$

Every isomorphism $\sigma_{s}$ can be extended in the natural way on the ring $\mathbf{Q}\left(\xi_{n}\right)[t]$, and obviously

$$
\sigma_{s}\left(F_{\chi}(t)\right)=F_{\chi}(t) .
$$

Since $F_{\chi}(t)$ is an element of $\mathbf{Z}\left(\xi_{n}\right)[t]$, we have $F_{\chi}(t) \in \mathbf{Z}[t]$.

\section{Main Results}

Let $N$ be an integer, $3 \leq N \leq 94$. By 2.4, 2.5 we can express the polynomial $d_{N}(t)$ as a product of integers polynomials $F_{\chi}(t)$. Let $K_{N}$ denote the number of these polynomials. We will enumerate them (for example according to the values of their degrees) and add the index $N$ so we have

$$
d_{N}(t)=\prod_{i=1}^{K_{N}} F_{N, i}(t) .
$$

Let $g_{N, i}$ be the product of all cyclotomic polynomials dividing $F_{N, i}$, and put $f_{N, i}=F_{N, i} / g_{N, i}$ for each $1 \leq i \leq K_{N}$. According to 2.1 the condition (2) holds if we find a prime $p=p(L, M, i, j)$ for each set of integers $L, M, i$, $j, 3 \leq L, M, L|N, M| N, 1 \leq i \leq K_{L}, 1 \leq j \leq K_{M}$ such that

$$
\operatorname{gcd}\left(f_{L, i}(t), f_{M, j}(1-t)\right)=1 \quad \text { over } \mathbf{Z} / p \mathbf{Z} .
$$

This was done using a personal computer. In most cases, (6) holds for polynomials $F_{L, i}(t), F_{M, j}(1-t)$, and some prime $p \leq 17$, so it is sufficient to compute only polynomials $F_{N, i}(t), F_{N, i}(1-t)$ modulo small primes. The calculation of polynomials $F_{N, i}(t), g_{N, i}(t), f_{N, i}(t)$, and $f_{N, i}(1-t)$ over $\mathbf{Z}$ is necessary only in a few cases (for example, if $\Phi_{3}(t) \mid F_{N, i}(t)$, because $\left.\Phi_{3}(t)=\Phi_{3}(1-t)\right)$. The relation (6) also holds in these cases for some prime $p, p \leq 17$.

Therefore, from our computation we obtain the following lemma.

3.1. Lemma. Let $L, M, i, j$ be integers, $3 \leq L, M, \operatorname{lcm}[L, M] \leq 94$, $1 \leq i \leq K_{L}, 1 \leq j \leq K_{M}$. Then there exists a prime $p \in\{2,3,5,7,11,17\}$ such that the polynomials $f_{L, i}(t), f_{M, j}(1-t)$ are relatively prime over $\mathbf{Z} / p \mathbf{Z}$.

The Main Theorem follows now immediately from 3.1, 2.6, 2.1, and 1.6. 
3.2. Theorem. Let $N$ be an integer, $2 \leq N \leq 94$. There exists an integer $L$ such that

$$
s(k, N) \equiv 0 \quad(\bmod l), \quad 0 \leq k \leq N-1,
$$

for each prime $l>L$ for which the first case of Fermat's Last Theorem is false for prime exponent $l$.

3.3. Remark. Let us try to find a value for the number $L$ in the last theorem. In our calculations we shall suppose that the polynomials $g_{n, i}$ have not been divided by cyclotomic polynomials $\Phi_{n}(t), n>45$. According to the proofs of 2.3 and 2.2 , the first condition for the number $L$ is that

$$
L>\left(\frac{1+\sqrt{5}}{2}\right)^{45^{2} / 3} \text {. }
$$

The second condition is that $L$ is greater than the largest prime dividing the number $c$ in (3). This certainly holds if $L$ is greater than the resultant of the polynomials $f(t), f(1-t)$ (it is known that the number $c$ divides this resultant-see [1, Lemma 20]).

We will find the rough upper bound of this resultant for the cases $N$ being a prime. In these cases we have

$$
\begin{gathered}
f(t)=\frac{d_{N}(t)}{g(t)}, \\
k=\operatorname{deg} f(t)=\operatorname{deg} f(1-t) \operatorname{deg} d_{N}(t)=\frac{\varphi(N)(N-2)}{2}=\frac{(N-1)(N-2)}{2} .
\end{gathered}
$$

Let $f(t)=\left(t-\alpha_{1}\right) \cdots\left(t-\alpha_{k}\right)$ over the field of complex numbers.

Each complex number $\alpha_{j}$ is a root of some polynomial $f_{\chi}(t)$, so we have

$$
\left|\alpha_{j}\right|^{N-2} \leq \sum_{i=0}^{N-3}\left|\alpha_{j}\right|^{i}
$$

hence $\left|\alpha_{j}\right|<2$.

It follows that

$$
R(f(t), f(1-t))=\prod_{i, j}\left(\alpha_{i}-\left(1-\alpha_{j}\right)\right)<5^{k^{2}} \leq 5^{(N-1)^{2}(N-2)^{2} / 4} .
$$

We have proved the next theorem.

3.4. Theorem. Let $N$ be a prime, $11 \leq N \leq 89$. Then

$$
s(k, N) \equiv 0 \quad(\bmod l), \quad 0 \leq k \leq N-1,
$$

for each prime $l>5^{(N-1)^{2}(N-2)^{2} / 4}$ for which the first case of Fermat's Last Theorem is false for prime exponent $l$.

\section{BIBLIOGRAPHY}

1. A. Granville and M. B. Monagan, The first case of Fermat's Last Theorem is true for all prime exponents up to $714,591,416,091,389$, Trans. Amer. Math. Soc. 306 (1988), 329-359.

2. M. Lerch, Zur Theorie des Fermatschen Quotienten $\frac{a^{p-1}-1}{p}=q_{(a)}$, Math. Ann. 60 (1905), 471-490. 
3. F. Pollaczek, Über den grossen Fermat'schen Satz, Sitzungsber. Akad. Wiss. Wien Abt. IIa 126 (1917), 45-49.

4. L. Skula, Fermat's last theorem and the Fermat quotients, Comm. Math. Univ. Sandilavli 41 (1992), 35-54.

5. B. L. van der Waerden, Moderne Algebra, Springer, Berlin, 1930-1931.

6. L. C. Washington, Introduction to cyclotomic fields, Springer-Verlag, New York, Heidelberg, Berlin, 1982.

7. A. Wieferich, Zum letzten Fermat'schen Theorem, J. Reine Angew. Math. 136 (1909), 293-302.

Družba 1289, 76824 Hulin, Czech Republic 